\title{
Concepções dos hipertensos sobre os fatores de risco para a doença
}

\section{Perceptions of hypertensive people on risk factors for the disease}

Mariana Carvalho Machado ${ }^{1}$

Cláudia Geovana da Silva Pires ${ }^{2}$

William Mendes Lobão ${ }^{3}$
${ }^{1}$ Saúde da Família,

Universidade do Estado da Bahia. Rua Silveira Martins 2555, Cabula. 41.150-000 Salvador BA.

enfa.mariana@gmail.com

${ }^{2}$ Escola de Enfermagem,

Universidade Federal da

Bahia.

${ }^{3}$ Universidade do Estado da

Bahia
Abstract This paper sought to verify the perceptions of hypertensive people in relation to the risk factors and their experience with high blood pressure in a Reference Center for Cardiovascular Diseases in the city of Salvador. Interviews were staged with 33 hypertensive people. This is a descriptive study, of a qualitative nature, supported by the discourse analysis proposed by Foucault. It was observed that risk factors were confused with hypertension complications. Nevertheless, when the approach changed from "risk factors" to "factors that can increase blood pressure," it was seen that the answers were more coherent with the risk factors classified by the VI Brazilian Policies on $\mathrm{Hy}$ pertension. Furthermore, it was also observed through the discourses that the perception of increase in blood pressure is directly related to experiences. Therefore, it is necessary that the guidance be transmitted as clearly as possible in order for the understanding to become an important facilitator for controlling the illness. This paper enabled the perception of the risk factors in the viewpoint of these people in such a manner as to supply clues for the interdisciplinary health team to promote healthcare based on the experiences and the socio-economic and cultural context in which these people are inserted.

Key words Hypertension, Knowledge, Risk factors, Nursing
Resumo Este estudo objetivou verificar as concepções dos hipertensos, os fatores de risco e suas vivências quanto ao aumento da pressão em um Centro de Referência em Doenças Cardiovasculares na cidade do Salvador-BA. Foram entrevistadas 33 pessoas hipertensas. Trata-se de um estudo descritivo, de cunho qualitativo, amparado na análise do discurso proposta por Foucault. Quanto aos conceitos de fatores de risco, percebeu-se que foram confundidos com as complicações da hipertensão, entretanto, quando a abordagem mudou de "fatores de risco" para "fatores que podem aumentar a pressão", as respostas foram mais coerentes e condiziam com os fatores de risco pontuados pelas VI Diretrizes Brasileiras de Hipertensão. Além disso, notou-se através dos discursos que a percepção de aumento da pressão está diretamente ligada às vivências e à história de vida de cada um. Sendo assim, é preciso que as orientações sejam transmitidas de forma mais clara possivel para que o entendimento possa servir de elemento facilitador para o controle da doença. Este estudo trouxe como ganhos a percepção dos fatores de risco na visão dessas pessoas de modo a fornecer pistas para a equipe de saúde interdisciplinar no intuito de promover o cuidado à saúde pautado nas vivências e no contexto socioeconômico e cultural nos quais estão inseridos.

Palavras-chave Hipertensão, Conhecimento, Fatores de risco, Enfermagem 


\section{Introdução}

A hipertensão arterial sistêmica (HAS) configura-se como uma doença crônica e um grave problema de saúde pública. Esta doença afeta bilhões de pessoas em todo o mundo e somente na Bahia foi responsável por 19.744 internações hospitalares de janeiro de 2009 a outubro de $2010^{1}$. A HAS também pode ser responsável pelo desenvolvimento de comorbidades a exemplo da doença coronariana, dos acidentes vasculares cerebrais (AVC), da insuficiência renal, das doenças vasculares periféricas, entre outras. As comorbidades consistem em complicações da hipertensão, diferentemente dos fatores de risco que são condições e comportamentos os quais contribuem com o desenvolvimento da doença hipertensiva ${ }^{2}$.

Devido à sua alta prevalência e morbidade (causa de 386 mortes diretas ${ }^{1}$ ) faz-se necessário uma maior atenção à prevenção a fim de evitar o desenvolvimento de novos casos ou que os existentes evoluam para quadros mais graves.

Para que a prevenção e a promoção da saúde sejam feitas de forma eficaz é necessário o conhecimento sobre a doença e dos fatores de risco que colaboram para o desenvolvimento da mesma ou das comorbidades associadas ${ }^{2}$.

O desenvolvimento da hipertensão não ocorre instantaneamente, há um conjunto de fatores que estão associados à sua evolução e agravo. Estes fatores são conhecidos como fatores de risco e, segundo as VI Diretrizes Brasileiras de Hipertensão Arterial, são: idade, sexo/gênero e etnia, fatores socioeconômicos, ingestão de sal, excesso de peso e obesidade, ingestão de álcool, genética e sedentarismo ${ }^{2}$. Além desses, outros autores acrescentam ainda o tabagismo e a não adesão ao tratamento $0^{3,4-6}$.

"Muitos fatores de risco para hipertensão são modificáveis, o que torna a hipertensão evitável na maioria dos casos ou com alta probabilidade de controle, se já presente"'. Etnia, idade, sexo e predisposição genética são fatores não modificáveis. E fatores ambientais e socioeconômicos são de difícil modificação, logo, a atenção do profissional com relação aos mesmos deve ser diferenciada. O sal, o álcool, a obesidade e o sedentarismo são passíveis de modificação a fim de reduzir o risco para hipertensão.

As pessoas hipertensas e a comunidade em geral devem ser informadas e educadas quanto a esses fatores; é necessário que todos saibam como os fatores de risco podem desencadear o aumento da pressão para que possam optar conscientemente por uma vida mais saudável.
Infelizmente, o número de hipertensos tratados ainda é pequeno diante da dimensão da doença. Apenas $50 \%$ dos hipertensos sabem de sua condição. Destes, metade não se trata e os outros $50 \%$ não tem a pressão sob controle $e^{3,7}$.

Um dos maiores problemas para este controle é a falta de adesão ao tratamento que ocorre em até $40 \%$ dos hipertensos, uma vez que além dos medicamentos são necessárias mudanças de hábitos ${ }^{3,7}$ que nem sempre são bem aceitas.

Além da falta de adesão ao tratamento, muitos estudos ${ }^{6,8,9}$ mostraram que a idade também se constitui como um fator de risco para hipertensão, pois ocorrem alterações na musculatura lisa e no tecido conjuntivo dos vasos sanguíneos devido ao envelhecimento ${ }^{5}$. Um estudo feito em Pelotas-RS com 1.968 pessoas comprova esta afirmação uma vez que mais da metade dos idosos com idades entre 60 e 69 anos são hipertensos, sendo que estes correspondem a $11,6 \%$ do total de participantes ${ }^{10}$.

Com relação ao sexo/gênero, as VI Diretrizes Brasileiras de Hipertensão consideram que o masculino é o mais afetado pela doença até os 50 anos de idade e as mulheres, ao produzirem hormônios femininos que são fatores de proteção, tendem a ter a incidência baixa antes da menopausa a qual aumenta a partir da sexta década de vida $^{2}$. Além disso, outros estudos também consideram que o sexo masculino é o mais afetado ${ }^{6,8}$.

A etnia negra predispõe os indivíduos a apresentarem níveis pressóricos mais elevados que a branca, evidenciando que aqueles têm maior propensão ao desenvolvimento da hipertensão ${ }^{5,10,11}$, além de terem mais chances de desenvolver as formas mais graves da doença e as patologias associadas ${ }^{12}$. Contudo deve-se levar em consideração a miscigenação da população brasileira que dificulta a classificação genérica da mesma.

Os fatores socioeconômicos podem estar associados ao controle dos níveis pressóricos e podem ser entendidos como nível de escolaridade e renda9. O nível de escolaridade é inversamente proporcional à hipertensão, ou seja, quanto maior o grau de instrução, menores os índices de hipertensão ${ }^{7}$. Este é um dado relevante uma vez que com o aumento do nível de instrução é mais fácil a compreensão das informações passadas a respeito da doença, das medicações, dos hábitos de vida e dos fatores de risco ${ }^{5}$. Contrastando com este dado um estudo realizado com 1.739 pessoas em uma capital brasileira não encontrou associações entre o nível de escolaridade e a hipertensão ${ }^{8}$.

A baixa renda e as más condições de vida estão associadas ao desenvolvimento da hiperten- 
são ${ }^{10}$. A baixa renda pode ser um fator determinante para a adesão ao tratamento, uma vez que dificulta o acesso a medicamentos, a alimentos adequados, a frequentar academias, entre outros ${ }^{9}$. Em contraponto, um estudo realizado em Salvador-BA, mostrou que tanto as pessoas aderentes quanto os não aderentes ao tratamento são de baixa escolaridade e baixa renda ${ }^{11}$. Logo, a associação da renda e da escolaridade com a HAS precisa ser mais bem estudada a fim de gerar dados concretos sobre sua relação com a hipertensão.

O sal contém em sua composição o sódio que é um potente estimulante cardíaco e, além disso, exerce atividades hipertensivas nos vasos sanguíneos periféricos ${ }^{13}$. Um estudo com pessoas negras hipertensas ${ }^{9}$, revelou que um terço dos entrevistados apresentava resistência para a redução de sal na alimentação, pois implica ter menos prazer com o consumo do alimento. Além disso, é importante ressaltar que no Nordeste o sal foi e é muito utilizado como conservante de alimentos, sendo então importante considerar a rotina dessas pessoas como um fator não só nutricional, mas socioeconômico e cultural. Estudos mostraram que os hipertensos têm consciência da importância da redução do sal para o tratamento uma vez que $62,5 \%$ e $74,3 \%$ dos entrevistados de tais estudos relataram fazer o controle do sal na alimentação $0^{5,7}$.

A obesidade e o excesso de peso associam-se com maior prevalência de hipertensão desde idades jovens. Na vida adulta o incremento de 2,4 $\mathrm{Kg} / \mathrm{m}^{2}$ no índice de massa corpórea (IMC) acarreta maior risco de desenvolver hipertensão, mesmo nos indivíduos fisicamente ativos ${ }^{2}$. Por isso, alguns autores comentam que a circunferência abdominal está mais associada à hipertensão do que a obesidade propriamente dita ${ }^{14}$.

A meta é alcançar um índice de massa corporal (IMC) inferior a $25 \mathrm{~kg} / \mathrm{m}^{2}$ e circunferência da cintura inferior a $102 \mathrm{~cm}$ para homens e $88 \mathrm{~cm}$ para mulheres, embora a diminuição de $5 \%$ a $10 \%$ do peso corporal inicial já seja capaz de produzir redução da pressão arterial ${ }^{15}$.

O sedentarismo está pronunciado nos $81,2 \%$ dos discursos de um estudo feito com 32 hipertensos, que disseram não fazer atividades físicas, logo se concluiu que o sedentarismo e a ingestão de gordura são os fatores menos controlados pelos hipertensos ${ }^{7}$. Outro estudo encontrou dados semelhantes: dos 1.039 indivíduos participantes, $70 \%$ eram sedentários ${ }^{16}$.

$\mathrm{O}$ alcoolismo está relacionado à hipertensão devido ao aumento da pressão arterial em 2 $\mathrm{mmHg}$ a cada $30 \mathrm{ml}$ de álcool etílico ingerido ${ }^{5}$.
Pelo fato da Sociedade Brasileira ser culturalmente adepta às bebidas alcoólicas, o consumo de álcool deve ser minuciosamente monitorado pelos hipertensos. Apesar deste dado, não foi encontrada relação entre a ingestão de bebidas alcoólicas e a hipertensão no estudo com 1.739 pessoas em uma capital brasileira ${ }^{8}$.

Outro fator de risco para hipertensão é o tabagismo. A nicotina presente no cigarro provoca o aumento do trabalho cardíaco, a disfunção do endotélio capilar, a liberação de catecolaminas e a hiper-reatividade vascular aumentando, consequentemente, a pressão arterial. Além disso, o fumo passivo, a terapia de reposição nicotínica e o uso da bupropiona (coadjuvante na cessação do hábito de fumar) devem ser considerados como causas em potencial de refratariedade ao tratamento anti-hipertensivo ${ }^{17}$. A prevalência do tabagismo é maior em homens e este fator somado à predisposição maior do sexo masculino de desenvolver hipertensão aumenta as chances dos homens tabagistas serem hipertensos ${ }^{18}$.

O fator genético está entre os fatores de risco pouco modificáveis para a $\mathrm{HAS}^{6}$. No estudo feito com 622 hipertensos em Salvador, observou-se que $56,4 \%$ dos pacientes brancos; $59 \%$ dos mulatos e $50,8 \%$ dos negros relataram haver história familiar de hipertensão $0^{12}$. Dado semelhante foi obtido no estudo feito com 32 hipertensos, onde $59,4 \%$ dos participantes disseram haver casos de hipertensão na família ${ }^{7}$. Sendo assim, a hereditariedade ou o fator genético é um forte indicador do desenvolvimento da hipertensão, por isso, os indivíduos com casos da doença na família devem estar atentos para monitorar o possível desenvolvimento da patologia.

Muito do tratamento da hipertensão deve ser baseado nos fatores de risco, alertando certos grupos para os pouco modificáveis (raça, idade, hereditariedade) e trabalhando junto ao hipertenso e sua família para mudar os que podem ser alterados (ingestão de sal e gordura, sedentarismo, obesidade, tabagismo, alcoolismo).

Neste aspecto, faz-se necessária uma ação conjunta entre a equipe de saúde, os familiares e os hipertensos na rede básica de saúde com destaque para a atuação do Enfermeiro na realização de atividades de educação em saúde para hipertensos em salas de espera, organização de caminhadas e cafés da manhã que visem, além de promover uma maior participação e inclusão social, fornecer informações precisas sobre os fatores de risco e efetivo controle da hipertensão.

Desta forma, traçou-se o objetivo geral: verificar as concepções dos hipertensos sobre os fa- 
tores de risco para a doença e suas vivências quanto ao aumento da pressão.

O conhecimento dos hipertensos sobre a doença e os seus fatores de risco é de grande relevância para que os profissionais de saúde delimitem o tratamento adequado, seja este medicamentoso ou não; ou até mesmo apenas para redução de danos, através da adoção de medidas que visem minimizar o impacto da hipertensão na vida de seus portadores, com a melhoria da condição de vida a fim de preservar os órgãos alvo (coração, vasos sanguíneos, cérebro e rins), levando em conta o seu perfil e as suas atitudes no momento das crises.

A equipe de saúde deve fornecer uma melhor educação e informação sobre a doença e os fatores de risco a fim de evitar o desenvolvimento da hipertensão e de quadros cardiovasculares mais complexos e facilitar a adesão ao tratamento por parte do hipertenso ${ }^{11}$.

\section{Métodos}

Este trabalho tem como base um estudo descritivo, de cunho qualitativo, cujo referencial teórico metodológico foi baseado nas falas dos sujeitos, fundamentado na análise do discurso proposta por Michel Foucault ${ }^{19}$. A coleta de dados foi feita através de um formulário semiestruturado de acordo com o objeto de estudo: concepções dos hipertensos sobre os fatores de risco para a doença.

Discurso, segundo Foucault ${ }^{19}$, é um conjunto de enunciados que se apóiam na mesma formação discursiva. Para o filósofo, nada há por trás das cortinas, nem sob o chão que pisamos. Há enunciados e relações, que o próprio discurso põe em funcionamento. Analisar o discurso seria dar conta de relações históricas, de práticas muito concretas, que estão "vivas" nele $e^{20}$.

O discurso é um método no qual o informante fornece as respostas para os questionamentos ao pesquisador, a depender dos tipos de perguntas feitas. Quando o formulário de entrevista é semiestruturado, há margens para respostas rápidas ou histórias orais de acordo com o cunho dos questionamentos que podem ser abertos ou fechados ${ }^{21}$. Segundo Vandresen ${ }^{22}$, "o discurso como objeto de análise é indispensável para quem quer compreender em que campo de relações entre saber e poder está inserido um sujeito".

Foucault compreende o discurso segundo o método genealógico como a ocorrência de uma relação entre o poder e o discurso onde os mes- mos são interdependentes, sendo que o discurso pode ser tanto instrumento do poder quanto efeito dele. Segundo o autor, o discurso produz o poder, conduz o mesmo, enrijece-o, porém também é capaz de corroê-lo. "A relação entre discurso e poder é acima de tudo uma relação produtiva, pois à medida que o poder se manifesta pelo discurso, este, por sua vez, produz mais poder"22.

Foram entrevistadas 33 pessoas hipertensas, de ambos os sexos, com idades maiores que 18 anos. A coleta de dados foi executada em um Centro de Referência em Doenças Cardiovasculares (CRDC), localizado em um bairro de classe média na cidade de Salvador (BA), onde todos os entrevistados estavam matriculados. A coleta foi feita durante 07 dias ( 24 de fevereiro a 04 de março), pela manhã, uma vez que o atendimento de enfermagem ocorre neste turno, e encerrou-se pelo critério de saturação dos dados. Os critérios de inclusão para a pesquisa foram:

- Hipertensão arterial diagnosticada há mais de 01 (um) ano, segundo os critérios das VI Diretrizes Brasileiras de Hipertensão ${ }^{2}$.

. Maiores de 18 anos.

. Com condições cognitivas de participar.

As três primeiras entrevistas foram realizadas para testar a efetividade e a objetividade do instrumento de coleta, sendo, então, descartadas da análise dos dados e as demais 30 foram utilizadas na pesquisa.

A entrevista foi gravada com o consentimento do entrevistado durante a aplicação do formulário, a fim de torná-la mais dinâmica, não desestimular o entrevistado pelo cansaço, não haver perda de tempo e, principalmente, garantir a fidedignidade dos dados.

O CRDC é uma unidade pública de saúde, de média complexidade especializada no atendimento de pessoas com afecções do sistema cardiovascular na cidade de Salvador (BA). Apresenta, aproximadamente, 27.000 clientes matriculados, conta com cerca de 47 profissionais de saúde e presta atendimento ambulatorial e serviço de apoio ao diagnóstico e tratamento (SADT) ${ }^{23}$.

$\mathrm{O}$ atendimento de enfermagem ocorre diariamente, porém somente pela manhã para as pessoas matriculadas no programa Hiperdia. Além deste, há a sala de aferição de pressão onde um enfermeiro coordena o trabalho dos técnicos de enfermagem e fornece orientações aos hipertensos e demais pessoas que busquem verificar a pressão.

A análise dos dados deu-se entre os meses de março e junho de 2010. Após a coleta de dados, as entrevistas foram transcritas ipsi literis para posterior análise. Foram realizadas leituras exaus- 
tivas e as respostas foram agrupadas segundo a qualidade das opiniões contidas, ou seja, segundo a concordância entre elas para facilitar a construção dos resultados e discussão.

Foram seguidos todos os preceitos éticos das pesquisas com seres humanos sendo respeitadas as determinações da resolução no196 de 10 de outubro de 1996 do Conselho Nacional de Saúde que regulamenta pesquisas com seres humanos ${ }^{24}$.

O trabalho foi aprovado pelo Comitê de Ética em Pesquisa da Sociedade Mantenedora de Ensino Superior da Bahia (CEP/SOMESB).

\section{Resultados e discussão}

\section{Características socioeconômicas dos entrevistados}

As pessoas entrevistadas compuseram uma população formada por 50\% (15/30) mulheres e $50 \%$ (15/30) homens, fato que comprova a inexistência de tendenciosidade dos relatos pelo gênero (masculino/feminino), as idades que prevaleceram foram aquelas acima de 56 anos [ $80 \%$ $(24 / 30)]$ e o grau de escolaridade mais prevalente foi o $1^{\circ}$ grau completo [53,3\% (16/30)]. Quanto à etnia, 46,7\% (14/30) dos entrevistados autodeclararam-se negros ou afrodescendentes; sobre a ocupação, 40\% (12/30) informaram estar aposentados(as) e a respeito da renda, a grande maioria, 73,3\% (22/30), afirmou ter renda familiar mensal variando entre 01 até 03 salários mínimos (Tabela 1).

\section{Fatores de risco segundo os hipertensos}

Quando questionados sobre o conceito de fatores de risco percebeu-se que a maioria das pessoas confundiu-os com as complicações advindas da doença, sendo que poucos deles conceituavam os fatores de risco, a maioria utilizou parâmetros de suas vivências cotidianas para definir tais fatores.

Os discursos decorrem da prática. E tudo está imerso em relações de poder e saber, que se implicam mutuamente ${ }^{22}$. Um trabalho feito com 32 hipertensos em Ribeirão Preto (SP) mostrou que a predisposição a outras doenças é considerada por eles consequência da hipertensão ${ }^{25}$. Dentre as consequências citadas, aquelas que se assemelham às encontradas neste estudo são: o derrame, o infarto e que "pode matar".

Dentre as respostas, foram mais frequentes aquelas referindo fatores de risco como sendo os
Tabela 1. Distribuição percentual das características socioeconômicas dos hipertensos entrevistados. Salvador-Bahia, março de 2010.

\begin{tabular}{|c|c|c|}
\hline & $\mathbf{n}$ & $\%$ \\
\hline \multicolumn{3}{|l|}{ Sexo } \\
\hline Masculino & 15 & 50,0 \\
\hline Feminino & 15 & 50,0 \\
\hline \multicolumn{3}{|l|}{ Idade em anos } \\
\hline 18 a 25 anos & 0 & 0 \\
\hline 26 a 35 anos & 0 & 0 \\
\hline 36 a 45 anos & 01 & 3,3 \\
\hline 46 a 55 anos & 05 & 16,7 \\
\hline 56 a 65 anos & 13 & 43,3 \\
\hline Acima de 65 anos & 11 & 36,7 \\
\hline \multicolumn{3}{|l|}{ Grau de escolaridade } \\
\hline Analfabeto & 01 & 3,3 \\
\hline $1^{\circ}$ grau incompleto & 06 & 20,0 \\
\hline $1^{\circ}$ grau completo & 16 & 53,3 \\
\hline $2^{\circ}$ grau incompleto & 02 & 6,7 \\
\hline $2^{\circ}$ grau completo & 04 & 13,3 \\
\hline $3^{\circ}$ grau incompleto & 0 & 0 \\
\hline $3^{\circ}$ grau completo & 01 & 3,3 \\
\hline \multicolumn{3}{|l|}{ Cor de pele autodeclarada } \\
\hline Negra/afrodescendente & 14 & 46,7 \\
\hline Pardo & 09 & 30,0 \\
\hline Branco & 01 & 3,3 \\
\hline Outra & 06 & 20 \\
\hline \multicolumn{3}{|l|}{ Ocupação } \\
\hline Nenhuma & 02 & 6,7 \\
\hline Carpinteiro & 01 & 3,3 \\
\hline Cozinheira & 01 & 3,3 \\
\hline Autônomo & 01 & 3,3 \\
\hline Motorista & 03 & 10,0 \\
\hline Eletricista & 02 & 6,7 \\
\hline Artesã & 01 & 3,3 \\
\hline Aposentado & 12 & 40,0 \\
\hline Doméstica & 01 & 3,3 \\
\hline Dona de casa & 06 & 20 \\
\hline \multicolumn{3}{|l|}{ Renda familiar mensal } \\
\hline Até 01 salário mínimo & 04 & 13,3 \\
\hline Entre 01 até 03 salários & 22 & 73,3 \\
\hline Acima de 03 salários & 02 & 6,7 \\
\hline Renda variável & 02 & 6,7 \\
\hline
\end{tabular}

Fonte: Dados obtidos através do formulário de coleta de dados da pesquisa.

acidentes vasculares cerebrais e os infartos ou a ocorrência dos mesmos, mencionados por $26,7 \%$ $(08 / 30)$ dos hipertensos cada um. Os trechos abaixo demonstram alguns dos relatos:

[...] Dá um derrame, qualquer coisa, né?[...] E6 É o derrame, o infarte. E10

As complicações da hipertensão foram lembradas pelos entrevistados por serem consequên- 
cias diretas do aumento descontrolado da pressão arterial, o que demonstra que eles conhecem o risco implícito na doença, mesmo desconhecendo seu conceito técnico-científico. Além disso, os mesmos seguem a "polícia discursiva" que, segundo Foucault, o que é dito "verdadeiro" reproduz-se nos discursos ${ }^{22}$. Assim, os entrevistados não saberem o conceito técnico de fator de risco, mas conhecerem os riscos propriamente ditos decorre do fato de que eles internalizam como verdade as informações que lhe são passadas e as que consideram relevantes saber.

A ingestão de sal e o aumento/alteração da pressão foram mencionados por $20 \%(06 / 30)$ dos entrevistados, cada um. As falas abaixo evidenciam algumas das afirmações:

Eu acho que fatores de risco da pressão é não comer sal, evitar o sal; [...] E5

A pressão tá muito alta [...] E7

A ingestão de gorduras e de bebidas alcoólicas aparece $16,7 \%(05 / 30)$ vezes nas respostas, exemplos abaixo:

Fatores de risco? [...] gordura, álcool [...]E15

[...] usar bebida alcóola, comer muita gordura. E17

Como se pode perceber, alguns dos relatos estão juntos na fala de um só entrevistado.

O tabagismo e a falta de autocuidado foram lembrados em 10\% (03/30) dos discursos, cada um:

Fatores de risco é a gente não vacilar com a pressão e cuidar da saúde, né? [...] E1

Fatores de risco? [...] cigarro [...] E15

Nos relatos acima, há bons exemplos de fatores de risco. $\mathrm{O}$ sal é um dos fatores, senão o mais importante, de aumento dos níveis pressóricos e foi citado apenas $20 \%$ das vezes. No estudo de Ribeirão Preto (SP), 19\% dos entrevistados citaram fatores alimentares como fatores de dificuldade de controle da pressão, sendo que $70 \%$ dessas citações foram quanto ao abuso do sal ${ }^{25}$.

Seria válido se o tabagismo emergisse mais vezes nas falas, contudo não se pode desprezar o valor destes poucos relatos, pois comprovam que alguns hipertensos sabem da interferência do fumo na pressão e não se pode esquecer que esses discursos têm muito a ver com os hábitos de cada um deles.

Ainda com certa relevância, foram lembrados a diabetes e a não adesão ao tratamento medicamentoso, cada item referido $6,7 \%(02 / 30)$ das vezes nas falas.

Nas últimas décadas a não adesão ao tratamento medicamentoso já vem sendo abordada como uma das preocupações dos profissionais de saúde ${ }^{26}$. Porém, pode ser mais considerada como um fator de risco para o desenvolvimento das complicações da hipertensão mais que da doença.

De acordo com Foucault, a unidade existente em um discurso não se deve a uma coerência visível e horizontal entre os objetos, porém se deve ao sistema que torna possível sua construção $0^{27}$. Neste contexto, os discursos construídos pelos hipertensos sobre os fatores de risco estão de acordo com o sistema que os cerca e com tudo aquilo que absorvem de informações transmitidas, seja pelos profissionais de saúde, por vizinhos, colegas, mídia, etc.

"A análise do discurso proposta por Foucault é um esforço de interrogar a linguagem o que foi efetivamente dito, sem a intencionalidade de fazer interpretações reveladoras de verdades e sentidos ocultos" ${ }^{\prime 2}$. Estes relatos não se devem à falta de conhecimento sobre a doença ou seus fatores de risco, pois ao serem questionados em suas próprias vivências, os entrevistados acima citaram fatores de risco. Além disso, o conceito da doença está intimamente ligado aos riscos que ela traz. Não há sentido oculto nos relatos destes hipertensos, apenas interpretações de informações recebidas da forma que acreditam ser a correta.

\section{Vivência dos hipertensos com fatores causadores da elevação da pressão}

De forma paralela, a questão seguinte investigou os mesmos conhecimentos, porém os questionamentos foram feitos com outros termos e enfocando naqueles vivenciados pelos entrevistados. Foram questionados sobre as situações, os comportamentos ou os fatores que podem elevar a pressão dos mesmos. As respostas obtidas foram muito mais objetivas e coerentes.

De 100\% (30/30) dos entrevistados, 40\% (12/ 30) afirmaram que o sal tem uma influência direta no aumento da sua pressão, contrastando com os $20 \%$ (06/30) entrevistados do tópico anterior que o consideram como um fator de risco:

Vamo ver se eu respondo certo: se eu comer sal demais, né? E3

Comida; o sal.[...] E5

O questionamento de E3 sobre sua resposta poder estar certa retoma o pensamento da "polícia discursiva" de Foucault ${ }^{22}$. Este entrevistado não responde diretamente segundo suas concepções, mas sim de acordo com o que lhe foi transmitido como correto.

Outros fatores muito relacionados ao aumento dos níveis pressóricos foram o aborrecimento $[36,7 \%(11 / 30)]$ e a preocupação $[16,7 \%$ 
$(05 / 30)]$. O primeiro foi indicado como um fator de risco por apenas 3,3\% (01/30) dos entrevistados quando questionados sobre tal, ao passo que quando questionados sobre a própria vivência, este número aumentou para 36,7\% (11/ 30). A segunda, não foi indicada por nenhum dos entrevistados como sendo um fator de risco para a pressão, no entanto, quando investigada a alteração da pressão pessoal, 16,7\% (05/30) das pessoas relataram preocupação como causa de aumento.

Aborrecimento. Eu não posso, se me aborrecer eu já sofro logo. Preocupação também. [...] E7

Os hipertensos acreditam muito que situações de estresse emocional, como as preocupações, podem elevar sua pressão ${ }^{9}$. No entanto, deve-se ressaltar que este quesito investigou as vivências de cada um com os fatores que aumentam a pressão. Desta forma, os fatores relatados têm mais influência na vida dos entrevistados do que outros com os quais eles não convivem ou não acreditam alterar sua pressão.

Outras situações de estresse emocional surgiram em 6,7\% (02/30) dos discursos, cada uma: ansiedade; problemas psicológicos; estresse e susto. Além de trabalho excessivo:

É o estresse, viu? Estresse, aborrecimento, o sal, só.[...] E17

Se eu tomar um susto. Ai ela... Aí sobe. [...] E18 [...] trabalhar muito também dá pressão alta [...] E22

As situações supracitadas não variaram do quantitativo de respostas comparando-se concepções sobre fatores de risco com as vivências. Apenas ansiedade, problemas psicológicos e trabalho excessivo surgiram como relatos novos, pois antes não haviam sido citados. Em um estudo realizado com 62 pessoas, percebeu-se que os hipertensos associam muito a doença aos "nervos"28.

Neste ponto percebe-se a heterogeneidade discursiva que, segundo Foucault, refere-se à dispersão de enunciados, remetendo a idéia de que os enunciados (unidades elementares do discurso) são, antes de qualquer coisa, acontecimentos ${ }^{27}$. Sendo assim, os acontecimentos nas vidas dos entrevistados tornam-se naturalmente seus enunciados, uma vez que são vivenciados por eles.

Com relação à ingestão de gorduras, 13,3\% (04/30) dos entrevistados associaram-na com o aumento da sua pressão. De forma que antes, $16,7 \%(05 / 30)$ deles haviam indicado a ingestão de alimentos gordurosos como fator de risco. Neste caso, não houve grande diferença entre os relatos de fatores de risco e os da vivência de cada um, entretanto, é importante perceber que houve um relato a mais quando o questionamento mudou.
Não comer tanta gordura, [...] E1

O uso das drogas lícitas, tanto as bebidas alcoólicas quanto o tabaco, também foram relatados por $13,3 \%(04 / 30)$ e $6,7 \%(02 / 30)$ dos entrevistados, respectivamente, como causas de elevação da pressão:

\section{[...]bebida; fumo. [...] E9}

Fazendo-se um paralelo com o quesito anterior, tem-se que não houve grande variação entre as opiniões dos entrevistados quanto a essas drogas serem fatores de risco, visto que o álcool foi citado por 16,7\% (05/30) dos entrevistados como fator e o tabagismo por 10\% (03/30). Porém, da mesma forma que aconteceu com os relatos para ingestão de gorduras, também ocorre um aumento nos relatos para as drogas.

Apesar de estarem muito presentes no cotidiano, as drogas lícitas acima não foram muito lembradas como fatores de risco nem associadas ao aumento da pressão na vivência das pessoas. No estudo com 1.739 pessoas não se encontrou correlação entre tabagismo, ingestão de bebidas alcoólicas ou sedentarismo e a ocorrência de hipertensão arterial $^{8}$. Devido a isto, as orientações sobre consumo de bebidas alcoólicas e uso de tabaco não sejam tão difundidas quanto àquelas voltadas à ingestão de sal, por exemplo.

Todavia, o álcool e o tabagismo não devem deixar de ser vistos como fatores de risco e é importante destacar que as opiniões quanto às drogas lícitas certamente surgiram por as mesmas estarem presentes nas vidas das pessoas que as citaram. Remetendo, novamente à concepção foucaultiana de que os enunciados são acontecimentos $^{27}$. E uma vez acontecendo na vida dessas pessoas, a associação com a doença é nitidamente mais clara para elas.

\section{Formas de percepção da alteração da pressão na vivência de cada um}

Atrelada à vivência da elevação da pressão causada pelos fatores supracitados, foi-lhes questionado sobre a forma como foi percebida essa elevação causada por tais fatores a fim de verificar as influências externas nas concepções dos entrevistados.

Através da análise dos discursos, percebe-se que o estresse emocional é um fator predisponente à percepção da elevação da pressão conforme relato de $36,7 \%$ (11/30) dos entrevistados:

[...] Ah, aumenta porque você fica nervosa, né? E você nervosa a tendência é a pressão subir. Não tem como descer, é aumentar, né? E4

É difícil para as pessoas se afastarem, evitarem ou ocultarem algo que está intrínseco à vida 
como a preocupação ${ }^{28}$. Este argumento esclarece a falta de explicação para a relação com a pressão. Muitos hipertensos podem considerar as preocupações como inevitáveis e só por serem preocupações já causam aumento na pressão.

Outros 10\% (03/30) dos entrevistados, disseram sentirem-se diferentes quando acreditam estar com os níveis pressóricos altos, a exemplo:

[...] Porque você sente um negócio diferente assim, aquela coisa diferente. Não é como quando você tá calminha. Aquela coisa calma... [...] E16

O descontrole da pressão é notado por meio de alterações físicas que lembram o hipertenso de fazer uso da medicação ${ }^{29}$. A percepção do aumento da pressão como "sensações diferentes" pode indicar que o hipertenso está prestes a sofrer alguma complicação como o infarto ou o AVC, tendo em vista que a hipertensão é uma doença que não costuma manifestar sintomas, sendo assim, deve-se dar uma atenção especial para essas sensações.

A percepção do aumento da pressão é muito pessoal e depende das vivências dos entrevistados, por isso, não se deve menosprezar nem desmerecer as informações por eles fornecidas. Foucault afirma que as pessoas têm conhecimentos independentes daqueles dos intelectuais ${ }^{27}$. Sendo assim, os hipertensos têm conhecimentos independentes dos profissionais de saúde e estes precisam atuar também nestes fatores vivenciados para que o hipertenso tenha uma atenção integral.

\section{Considerações finais}

Os entrevistados apresentam concepções interessantes sobre os fatores de risco. Apesar de confundirem os mesmos com as comorbidades, este fato demonstra que os hipertensos conhecem o risco implícito na doença. Além disso, os hipertensos entrevistados conhecem os fatores de ris- co, mas nem sempre pelo termo técnico "fator de risco" empregado pelos profissionais de saúde e isto fica comprovado com a mudança da abordagem para "fatores que podem aumentar a pressão". Após a mudança nos termos, os entrevistados passam a relatar fatores que, de fato, são fatores de risco para a hipertensão.

Sobre a percepção do aumento da pressão, este estudo mostra que as mesmas são extremamente particulares para cada pessoa hipertensa, ou seja, cada uma delas acredita que sua pressão aumenta por fatores diversos e distintos.

Sendo assim, é preciso que as orientações sejam dadas de forma mais clara possível para que o entendimento aconteça, bem como as mesmas devem estar conectadas com a vida da pessoa e a vivência que ela tem com a HAS, de forma específica e personalizada. As pessoas precisam saber diferenciar a causa da consequência. Uma medida que se mostra eficaz é utilizar a linguagem da própria população para passar essas informações e evitar os termos técnicos. Ou seja, a população conhece os riscos que tais fatores agregam, contudo desconhece os nomes atribuídos aos mesmos pelos profissionais de saúde.

Os entrevistados têm autoconhecimento suficiente para saber o que desencadeia o aumento dos próprios níveis pressóricos e esta é outra base importante para a atuação da equipe de saúde. Conhecendo-se a história de cada pessoa é possível atuar nos fatores que não são perceptíveis em uma entrevista simples. A necessidade da criação de um vínculo dos hipertensos com a equipe é essencial para atuação e resolução/atenuação desses fatores.

Este trabalho proporcionou a percepção dos fatores de risco sob a ótica dos hipertensos, o que eles pensam a respeito e acreditam serem os fatores que interferem em sua pressão. Trazendo assim, uma rica fonte de informações para equipe de saúde saber como lidar/atuar junto a essas pessoas. 


\section{Colaboradores}

MC Machado trabalhou na concepção, no delineamento e na interpretação dos dados e na redação final do artigo. CGS Pires trabalhou na concepção e na revisão crítica, e aprovou a versão a ser publicada. WM Lobão trabalhou na concepção, revisão crítica e aprovou a versão a ser publicada.

\section{Referências}

1. Brasil. Ministério da Saúde (MS). Secretaria de Gestão Estratégica e Participativa. Departamento de Informática do SUS (DATASUS). Informações de saúde: dados de morbidade. Brasília: MS; 2010.

2. Sociedade Brasileira de Cardiologia - SBC; Sociedade Brasileira de Hipertensão - SBH e Sociedade Brasileira de Nefrologia - SBN. VI Diretrizes Brasileiras de Hipertensão. São Paulo: 2010. [documento da Internet]. Disponível em: http://www.saude.al.gov.br/ files/VI_Diretrizes_Bras_Hipertens_RDHA.pdf

3. Lessa I. Impacto social da não-adesão ao tratamento da hipertensão arterial. Rev. Bras. Hipertens. 2006; 13(1):39-46.

4. Gusmão JL, Mion Júnior D. Adesão ao tratamento conceitos. Rev. Bras. Hipertens. 2006; 13(1):23-25.

5. Pessuto J, Carvalho EC. Fatores de risco em indivíduos com hipertensão arterial. Rev. Latino-Am. Enfermagem 1998; 6(1):33-39.

6. Mano GMP, Pierin AMG. Avaliação de pacientes hipertensos acompanhados pelo Programa Saúde da Família em um Centro de Saúde Escola. Acta Paul. enferm 2005; 18(3):267-275.

7. Simonetti JP, Batista L, Carvalho LR de. Hábitos de saúde e fatores de risco em pacientes hipertensos. Rev. Lat. Am. Enfermagem 2002; 10(3):415-422.

8. Jardim PCBV, Gondim MRP, Monego ET, Moreira HG, Vitorino PVO, Souza WKSB, Scala LCN. Hipertensão Arterial e alguns fatores de risco em uma capital brasileira. Arq. Bras. Cardiol. 2007; 88(4):452457.

9. Pires CG da S, Mussi FC. Crenças em saúde para o controle da hipertensão arterial. Cien Saude Colet 2008; 13(2):2257-2267.

10. Costa JSD da, Barcelos FC, Sclowitz ML, Sclowitz IKT, Castanheira M, Olinto MTA, Menezes AMB, Gigante DP, Macedo S, Fuchs SC. Prevalência de hipertensão arterial em adultos e fatores de risco associados: um estudo de base populacional urbana em Pelotas, Rio grande do sul, Brasil. Arq. Bras. Cardiol. 2007; 88(1):56-65.

11. Lessa I, Fonseca J. Raça, aderência ao tratamento e/ou consultas e controle da hipertensão arterial. Arq. Bras. Cardiol. 1997; 68(6):443-449.

12. Noblat ACB, Lopes MB, Lopes AA. Raça e lesão de órgãos-alvo da hipertensão arterial em pacientes atendidos em um ambulatório universitário de referência na cidade de Salvador. Arq. Bras. Cardiol. 2004; 82(2):111-115.

13. Smeltzer SC, Bare BG. Histórico e tratamento de pacientes com hipertensão In: Smeltzer SC, Bare BG, Hinkle JL, Cheever KH. Tratado de enfermagem médico-cirúrgica. 10 $0^{\text {a }}$ ed. Rio de Janeiro: Guanabara Koogan; 2006. p. 904-916.

14. Hasselmann MH, Faerstein E, Werneck GL, Chor D, Lopes CS. Associação entre circunferência abdominal e hipertensão arterial em mulheres: estudo prósaúde. Cad Saude Publica 2008; 24(5):1187-1191.

15. Brasil. Ministério da Saúde. Secretaria de Atenção à Saúde. Departamento de Atenção Básica. Cadernos de Atenção Básica no 15: Hipertensão Arterial Sistêmica. Brasília: MS; 2006. [acessado 2012 abr 15]. Disponível em: http://www.prosaude.org/publicacoes/ diversos/cad_AB_hipertensao.pdf 
16. Souza LJ de, Neto CG, Chalita FEB, Reis AFF, Bastos DA, Filho JTDS, Souza TF, Côrtes VA. Prevalência de Obesidade e Fatores de risco cardiovascular em Campos, Rio de Janeiro. Arq. Bras. Endocrinol. Metab. 2003; 47(6):669-676.

17. Moreno Júnior H, Toledo JCY, Fonseca FAH. Hipertensão refratária e tabagismo. Rev. Bras. Hipertens. 2004; 11(4):256-261.

18. Nunes Filho JR, Debastiani D, Nunes AD, Peres KG. Prevalência dos fatores de risco cardiovascular em adultos de Luzerna, Santa Catarina, 2006. Arq. Bras. Cardiol. 2007; 89(5):319-324.

19. Foucault MA. Arqueologia do saber. Rio de Janeiro: Forense; 1986.

20. Fischer RMB. Foucault e a análise do discurso em educação. Cad. Pesqui. 2001; 114(Nov.):197-223.

21. Polit DF, Back CT, Hungler BP. Fundamentos de pesquisa em enfermagem: métodos, avaliação e utilização. 5a ed. Porto Alegre: Artmed; 2004.

22. Vandresen DS. O discurso na arqueologia e genealogia de Michel Foucault. [texto da Internet] [acessado 2012 abr 15]. Disponível em: http://www.diaadiaeducacao. pr.gov. br/diaadia/diadia/arquivos/File/conteudo/ artigos_teses/FILOSOFIA/Artigos/Daniel_Salesio_ Vandresen.pdf

23. Brasil. Ministério da Saúde (MS). Secretaria de Gestão Estratégica e Participativa. Departamento de Informática do SUS (DATASUS). Cadastro Nacional de Estabelecimentos de Saúde (CNES). Brasília: MS; 2010. [acessado 2012 abr 15]. Disponível em: http://cnes.datasus.gov.br/cabecalho_reduzido. asp? VCod_Unidade $=2927400004235$

24. Brasil. Resolução nº 196, de 10 de outubro de 1996. Aprova as diretrizes e normas regulamentadoras de pesquisas envolvendo seres humanos. Diário Oficial da União 1996; 10 out.
25. Péres DS, Magna JM, Viana LA. Portador de hipertensão arterial: atitudes, crenças, percepções, pensamentos e práticas. Rev Saude Publica 2003; 37(5): 635-642.

26. Leite SN, Vasconcellos MPC. Adesão à terapêutica medicamentosa: elementos para a discussão de conceitos e pressupostos adotados na literatura. Cien Saude Colet 2003; 8(3):775-782

27. Moraes AT. O discurso em Foucault: noções para uma prática jornalística. XII Congresso de Ciências da Comunicação na Região Centro-Oeste; 27-29 maio 2010; Goiânia, Goiás.

28. Gandarillas MA, Câmara SG, Scarparo H. Estressores sociais da hipertensão em comunidades carentes. Psicol. Reflex. Crit. 2004; 18(1):62-71.

29. Machado LRC, Car MR. Dialética do modo de vida de portadores de hipertensão arterial: o objetivo e o subjetivo. Rev. esc. enferm. USP 2007; 41(4):573580 .

Artigo apresentado em 26/03/2011

Aprovado em 18/10/2011

Versão final apresentada em 03/11/2011 